\title{
A Statistical Mechanics Approach to the Study of Energy Use Behaviour
}

\author{
Godwin Osabutey $\mathbb{D}^{1},{ }^{1}$ Alex Akwasi Opoku $\mathbb{D}^{1},{ }^{1}$ and Samuel Gyamfi ${ }^{2}$ \\ ${ }^{1}$ Mathematics and Statistics Department, University of Energy and Natural Resources, P. O. Box 214, Sunyani, Ghana \\ ${ }^{2}$ Energy and Engineering Department, University of Energy and Natural Resources, P. O. Box 214, Sunyani, Ghana \\ Correspondence should be addressed to Alex Akwasi Opoku; alex.opoku@uenr.edu.gh
}

Received 12 November 2019; Accepted 23 March 2020; Published 1 May 2020

Academic Editor: Abdel-Maksoud A. Soliman

Copyright (C) 2020 Godwin Osabutey et al. This is an open access article distributed under the Creative Commons Attribution License, which permits unrestricted use, distribution, and reproduction in any medium, provided the original work is properly cited.

\begin{abstract}
This paper proposes a statistical mechanical model for the energy conservation behaviour of energy users. The model was inspired by the multipopulation Curie-Weiss model and the discrete choice with social interaction model. We demonstrate that the model integrates the sociopsychological, the economic, and the energy technologist viewpoints to energy management, allowing us to subject these perspectives to an empirical study.
\end{abstract}

\section{Introduction}

Energy is one of the most important drivers of the economy of every country. Often, managing the energy resources has been a problem. Electrical energy plays an indispensable role in undertaking most of our daily activities from refrigeration, cooking, lighting, entertaining to powering of machines and record keeping in the industrial and commercial sectors [1]. Electrical energy serves as a crucial resource for productive and quality education, efficient healthcare delivery, transportation systems, and the exploration of minerals such as iron and gold. These serve as the building block on which every sector of a nation's economy thrives for development [1]. The efficient use of resources available at a given time by the energy end users has also been a challenge. The demand for energy around the world keeps on increasing, and the existing traditional forms of energy such as the fossil fuels and coal, which are not renewable sources of energy, are struggling to keep up with this demand over time. Moreover, the regular usage of the fossil fuels has a negative impact on global warming and the ecosystem. Adaptation of a better and dynamic demand response program which promotes energy conservation practices among energy users is highly sought after now than ever. Economic-engineering studies have attempted to close this gap in energy efficiency through the adoption of different technologies [2]. A model that can simultaneously capture group dynamics, body use, cognitive processes, and human-machine interactions is needed. To date, progress towards such a model has been limited by the theoretical preferences of the various disciplines involved in energy research [2].

The main interest here is to import ideas from discrete choice theory and statistical physics to develop models for energy conservation decision-making. Here, our focus is on a model that captures how individuals' decision about energy conservation is influenced by their socioeconomic attributes and the choices of others. This work is structured as follows: Section 2 gives an overview of existing models and theories in relation to human behaviour and energy use. In Section 3, we propose a model from discrete choice theory as a model for interdependent energy conservation decision-making. Section 4 discusses a more generalised form and multipopulation version of the Curie-Weiss model and provides an estimation procedure suitable for the model. Conclusion of the work is found in Section 5.

\section{Modelling of Energy Use Behaviour}

Modelling of individual behaviour has always been a challenge in the context of energy conservation. There are various 
schools of thought here. These are the economists, energy technologists/analysts, and sociopsychologists schools of thought. Their approaches to modelling human energy use and behaviour will be discussed below, and we will propose a model which can incorporate all the three approaches later. This section will begin by discussing the economists and energy technologists approach, and this will be followed by the sociopsychologist approach. These approaches are qualitative and form the theoretical framework of this study.

2.1. Economists Approach. The price of energy and income of individuals is the main determinants of individuals energy conservation behaviour in economics studies. The theory of utility maximization and consumer rationality in microeconomics modulates consumer's demand. This theory is derived from the concept that consumers always want to maximize their utility at the least possible cost, i.e., consumers try to weigh all other alternatives and actions to see the one that will maximize their utility/satisfaction [3]. In the economics viewpoint, rationality is defined as a fundamental axiom which control consumer behaviour. Consumer energy conservation decisions must be rational, and effective policies such as labels on electrical appliances and energy demand response programs (efficiency methods) are liable to weaken the economic theory of rationality by constraining consumers of want for options [4].

In [4], the authors examined the disparities between consumer rationality and other alternatives to energy efficiency, and they found it useful to describe the link between consumer rationality and energy demand behaviour. The first viewpoint considered in [4] is attributed to ideologies from neoclassical economics. According to a neoclassical economic viewpoint on consumer rationality and energy demand behaviour as discussed in [4], the consumers of energy and suppliers/producers have a fixed preference that they both endeavour to fulfil by means of market mediation. The underlined preferences of consumers turn out to influence their decisions to accept or reject energy-efficient appliances and equipment. These usually reflect a consumer's rational and relevant evaluation of costs and benefits from a certain technology or practice.

Economists always base their points of view on the notion that individuals react to the "performance of the market system in terms of informal arguments based on intuitive notions of rationality and preference" [5]. In this case, if rational theory of choice is a literal system of describing consumer behaviour, then energy consumers, producers, and policy makers face the challenge of solving complex optimization problems daily. These problems are not just for a daily cost minimization but also becomes an optimal control problem and stochastic dynamic problems $[4,5]$.

2.1.1. Limitation to the Economists Approach. The economic theory of utility maximization and consumer rationality is used as a model for influencing consumers' demand with price. An experiment conducted by psychologists has shown that individuals do not consistently make rational decisions [6] and their behaviour is not consistent with the standard model of rational choice in economics [4]. Some of the factors include time inconsistency, reference dependence, and bounded rationality. The economic theory of decisionmaking is incapable to fully explain human behaviour, to be specific behaviours related to residential energy use. The theory of bounded rationality suggests that "individuals employ heuristics" when making choices instead of the standard "strict rigid rules of optimization" [6].

2.2. Energy Technologists Approach. The second view point discussed in [4] is also related to consumer rationality and other alternatives to energy efficiency and attributed to energy technologists and behavioural scientists. Energy technologists and behavioural scientists assert that individuals' choices for their preferences do not minimize their costs of obtaining energy services. In the view of technological analysts, consumers are not having accurate knowledge regarding energy-efficient technologies and lack the ability to make accurate decisions even when they are given full and complete information [4]. Making accurate and precise decisions is based on relevant technical skills which are possessed by experts and specialists in economics, mathematics, and energy-related disciplines. Making correct choices always as supposed by economists is highly impossible, since there will be a need of high-speed computers to run complex optimization problems on energy technologies [4].

The work in [6] also contributes to understanding energy efficiency and stresses on the importance of human behaviour as a factor that is always ignored. The author discusses the following three basic routes that can lead to energy savings:

(1) Replacing the existing housing stock with low-energy buildings designed primarily to minimize heating and cooling loads [6]

(2) Developing energy-efficient domestic equipment [6]

(3) Promoting and achieving "energy-conscious" behaviour among end users [6]

Energy-efficient methods mostly follow the first two route, i.e., the design of buildings and energy-efficient appliances. The "physical-technical economic model" (PTEM) is one of the dominant models in energy analysis. The PTEM proposes that the energy conservation in buildings/households relies almost always on the engineering design (physical characteristics) of buildings and the efficiency of technological appliances. This model overlooks and considers human behaviour as an insignificant factor to reducing peak demand in the residential sector of energy consumption.

The PTEM focuses on energy pricing, technological tools, and improvement and considers social actions and noneconomic factors as an insignificant aspect of consumption [7]. This is clearly contrary to what happens in the real world. Household energy consumption varies, and this can be attributed to variations in engineering design and economic factors, demographic characteristics (gender, employment status, household size, race, etc.), and the behaviour of the household members. 
2.2.1. Limitation to the Energy Technologists Approach. The PTEM focuses on energy pricing, technological tools, and improvement and considers social actions and noneconomic factors as an insignificant aspect of consumption. On the contrary, social behaviours and noneconomic factors are significant in the study of human energy consumption activities [7].

2.3. Sociopsychological Approach. Research has shown that consumers do not always act rationally as suggested by economist. Individual behaviour is bounded by time, and rationality and individuals are reference dependent [6]. According to $[8,9]$, the consumers of energy do not just consider the minimization of energy cost in their day to day activities. Energy users are also consumers and therefore show higher preferences for things such as comfort and appearance than the monetary benefits obtained from using energy-efficient appliances. Consumers are in a social group and therefore influenced by the things they hear from neighbours or family more than information from distant energy experts.

As stated earlier, the theory of bounded rationality suggests that "individuals employ heuristics to make a decision rather than a strictly rigid rule of optimization" [6]. This is because consumers do not have the ability to compute the utility of all alternatives and the complexity of situations. Researchers have been stressing the importance of studying residential energy consumption from a social context. It is believed that despite the fact that encouraging changes in individuals' behaviour towards energy conservation is relevant, analysis from a social perspective will also provide a broad framework to understand residential energy conservation behaviours of individuals [3].

2.3.1. Theory of Reasoned Action. The model proposed by Icek Ajzen which is known as the theory of reasoned action makes an attempt to explain human behaviour [10]. According to [11], behavioural intention is simply what one intend to do or not to do, and it is the most appropriate determinant of behaviour. Behavioural intention is explained by the attitude (A) and subjective norm (SN) of an individual. Attitude is simply an individual's evaluation of behaviour, whereas subjective norm refers to the influence from the society or surrounding on an individual to accept or not to accept a certain norm or belief. In [10], the author treats the likelihood of response by an individual as three self-standing notions, namely belief, attitude, and intention. The work in [12] also indicates that for any behaviour, individuals have several beliefs but have the ability to make use of only few at any given instant. Beliefs about likely consequences of attitude and its evaluations are the major determinants of attitude $[10,11]$. According to [10], it is the case that individuals establishes/builds beliefs about an object by associating it to an underlying characteristics or attributes of the object in question. An example of how individuals form beliefs is as follows; after a careful study of the exam result, it is obvious that the students (object) in the class learned very hard or copied each other or brought in foreign materials or stole the questions (attributes) [10]. These attributes are evaluated to be positive or negative by an individual concerning the object. Hence, individuals immediately develop an attitude towards the object. It is clear from these points that attitude is a result of beliefs about an object, and how it is evaluated by an individual. Suppose an individual has $n$ beliefs $\left(b_{1}, b_{2}, \cdots, b_{n}\right)$ and $n$ subjective evaluations $\left(e_{1}, e_{2}, \cdots, e_{n}\right)$ of the beliefs. It follows from $[10,13]$ that the attitude $A$ of the individual is proportional to the sum of the product of the beliefs and their evaluations, i.e.,

$$
A \propto \sum_{i}^{n} b_{i} e_{i}
$$

Thus, attitude is proportional to the scalar product of the vectors $b=\left(b_{1}, b_{2}, \cdots, b_{n}\right)$ and $e=\left(e_{1}, e_{2}, \cdots, e_{n}\right)$ of beliefs and subjective evaluations, respectively.

As indicated above, behaviour is explained by both the attitude and subjective norm of individuals. Subjective norm is simply how an individual perceive others approval about his or her behaviour. The strength of normative belief $s_{i}$, for $i=1,2, \cdots, n$, is concerned with the evaluation made by an individual's reference group, peers, colleagues, family, etc. relating to the approval or disapproval for the individual to hold belief $b_{i}$. Subjective norm is determined by beliefs or thoughts others esteem as important about specific things one should do, and how much one is motivated to comply $\left(m_{1}, \cdots, m_{n}\right)$ with others on beliefs $\left(b_{1}, b_{2}, \cdots, b_{n}\right)$ [13]. Subjective norm is directly proportional to the sum of the products of the strength of each normative belief $\left(s_{i}\right)$ and an individual's motivation to comply $\left(m_{i}\right)$ to the belief $b_{i}$ across the $n$ different beliefs $[10,11,13]$. This can be mathematically expressed as

$$
S N \propto \sum_{i}^{n} s_{i} m_{i}
$$

To properly explain behaviour, the theory of reasoned action proposes that behaviour $(B)$ is approximated by behavioural intention $(B I)$, and $B I$ is proportional to the sum of attitude and subjective norm. Thus,

$$
B \approx B I=k_{1} A+k_{2} S N=k_{1} \sum_{i=1}^{n} b_{i} e_{i}+k_{2} \sum_{i=1}^{n} s_{i} m_{i} .
$$

2.3.2. Theory of Planned Behaviour. The theory of planned behaviour is also a sociopsychological theory which aims at explaining human behaviour [13]. This theory is an extension to the theory of reasoned action which looks at the possible risky prediction that can be made with the theory of reasoned action [11]. The theory of planned behaviour extends the theory of reasoned action by adding perceived behavioural control. Perceived behavioural control describes the extent of control individuals think they have over their behaviour, and it comes as an additional factor to behavioural intention. It is usually measured by things such as "passing the exam is under my control" and "it is easy for me to pass the exam" [11]. According to [11], perceived 
behavioural control is included in behavioural intention; hence, behavioural intention can be used directly to predict behavioural achievement. The theory says that the most appropriate determinant of one's actual behaviour is simply the motive an individual possesses to act in a certain manner. The core to the theory of reasoned action discussed above is intention. It can be noted that intention is central to this theory of behaviour as well. It is assumed that intention plays the role of capturing motivational factors that influences the actual behaviour of an individual $[10,13,14]$. Given the nature of a problem, behavioural intention is a description of the willingness of individuals to attempt and an indication of extensive effort willing to exert in order to adopt a behaviour $[10,13,14]$.

2.3.3. Goal-Framing Theory. The goal-framing theory deals with cognitive process and studies environmental behaviours. According to the work in [15], the goal-framing theory is motivated by studies revolving around cognitive social psychology and aims at influencing one's goals during cognitive processes. Cognitive processes consider the mental function of the mind related to logical reasoning and evaluations. For the theory to work, an individual is given a prior knowledge about certain innovations. Then, the receiver of the knowledge forms an attitude towards the innovation, decides to reject or approve of the innovation based on the knowledge acquired, applies it, and confirms a decision. The framework identifies three goals that are relevant to the study of environmental behaviour. These goals are the normative, hedonic, and gain goals, which are mostly relied on for proenvironmental behaviour studies [15]. In regard to this theory, normative goal frame is defined to be the motivation an individual has "to act appropriately." When an individual is obsessed with this goal, then an individual has the tendency to act "according to one's own or others norm's" in the most appropriate way and kindled to portray proenvironmental behaviour [15]. An individual inclined to hedonic goal is concerned with things that enable him or her to "feel better right now." These include obsession in seeking personal pleasure, contentment, or arousing one's personal interest. Individuals with this goal are very sensitive to things that affect their satisfaction or pleasure [15]. An individual falling under the gain goal frame is one with the motivation to "guard and improve one's resources" [15]. The framework discussed above is mostly used in analysing and developing policies and making decisions concerning proenvironmental behaviours. Considering the three goal frames discussed, an effective strategy to foster interest in proenvironmental behaviours will be strengthening normative goals to reduce the adverse impact of one's behaviour on nature [16]. By doing so, individuals would behave appropriately even when hedonic or gain goals are focal. From this perspective, the normative goal seekers' behaviour is consistent with the reasoned action behaviour if an individual's environment is taken to be the reference group of the individual, and the reasoned action behaviour is dominated by the subjective norm.

Sociopsychological research have indicated that human behaviour can better be explained by many factors (e.g., norms, beliefs, values). Consider an individual with higher value for the environment. This individual will more likely respond to environmental information than prices, while an individual with higher value for energy security (blackout) will respond to information relating to practices that will ensure no blackout than prices and environment [6].

The authors in [17] investigated the belief that being a witness to the behaviour of others significantly influence the behaviour of an individual, and it can lead to people believing in things that are untrue, saying things that are false, or doing things that are wrong. In their work, they conducted two studies with the first study focusing on exploring respondents' stated reasons for engaging in energy conservation [17], while the second study provided an initial test of the actual factors influencing participants' conservation behaviour [17]. In the first study, participant indicated that the behaviour of their neighbours (descriptive norm) had the least influence on them to conserve energy. Result from the second study indicates that descriptive norms is the most influential factor to consider concerning individual energy conservation behaviour. They also found out that the environment and moral obligation to the society are the most important factors to practicing energy conservation. In fact, results from the study suggest that energy users do not know what actually motivates them to practice energy conservation and would not be able to forecast the most effective approach to conservation. It was also noted that normative influence is a powerful but underrated form of all social influence [17].

2.3.4. Limitation to the Sociopsychological Approach. In energy-related decisions, the sociopsychological models discussed above provides qualitative interpretations to individuals' behaviour. It lacks the ability to interpret the effect of social interaction among group of individuals based on certain socioeconomic attributes.

2.4. Logistic Regression Approach. Logistic approach is a wellknown technique in predicting the behaviour of individuals. A study done in [14] employed ideas from the goal-framing theory with the interest to determine the most dominant goal in energy efficiency practices among individuals with knowledge about energy conservation. The utility company Latvenergo, in Latvia, was used as the survey area. The utility company Latvenergo is the main supplier and distributor of energy in Latvia. The main idea behind their study was to delve into the attitude or behaviour of people that are well vest in energy efficiency to investigate how they form attitude towards energy efficiency practices. A correlation analysis was carried out to study the relationship between the response of participant being actively engaged in energy-saving activities and all other answers of the questionnaire. The logistic approach imported by the researchers was intended to identify a linear combination of factors that could possibly explain the behaviour of the staff of Latvenergo to practice or not to practice energy conservation behaviours. The logistic function used in the work is given by

$$
\log \left(\frac{P(X)}{1-P(X)}\right)=\beta_{0}+\beta_{1} x_{1}+\beta_{2} x_{2}+\cdots+\beta_{k} x_{k} .
$$


Here, $P(X)$ represents the probability for individual to engage in energy savings activity, $\beta_{k}$ is the coefficient of the $k^{\text {th }}$ independent variable $x_{k}$, and $\beta_{0}$ is the constant in the model.

Their result from the correlation analysis indicated that respondents who are actively practising energy conservation behaviours and those that are not, expressed, most of the time, normative view. It was also observed from their fitted regression model that there exists no one clear goal dominating individual's motivation in Latvenergo. The authors made use of a final model they called "energy efficiency motivation," this model combines all the three goal frames and with other variables. This final model was able to explained 51.3685\% deviance in the data and $13.0465 \%$ of adjusted deviance.

According to the authors, the final model had a lower adjusted deviance when compared with similar studies [18, 19]. They believe that the broad survey setting could be a factor and suggest a methodical improvement which will narrow down to a specific energy efficiency practice such as the usage of LED appliances.

2.4.1. Limitation to the Logistic Model Approach. The logistic model studied above considers only socioeconomic attributes of individuals and makes no use of interaction among individual. Individuals are in a community and therefore influenced by the decisions of others within their reference group and beyond.

The studies conducted above bring to light various perspectives of energy efficiency strategies and implementations in support of demand response programs. The studies discussed so far are all qualitative with the exception of Section 2.4 and therefore forms a theoretical background of this paper. These theories and models will culminate into a quantitative model which will be used to analyse individual's energy use behaviour in a community. The interest from the above viewpoints is a mathematical model that incorporates all these ideas and how individuals' decisions about energy conservation are influenced by their socioeconomic attributes and interaction.

\section{Quantitative Model for Human Behaviour}

A quantitative model that integrates group interactions, body use, cognitive processes, and human-machine interactions will be desirable. Progress towards such a model has been limited by the theoretical preferences of the various disciplines involved in energy research [2].

3.1. Modelling of Decision-Making and Energy Use. In the work of [2], the authors emphasized that theoretical approaches to decision-making have two main roles:

(1) To explain behaviour and identify important behavioural drivers for interventions to target

(2) To provide a framework for empirical research on the impact of these interventions

Intervention in their work means any regulation, policy, program, measure, activity, or event that aims to influence behaviour [2]. According to [7], social processes or influences are quite complex and always ignored when modelling energy conservation. Individual behavioural study is good, but individuals are in a society of which collective behaviour has influence on their behaviour and choices. Families and large social groups are supposed to be considered in the analysis of energy conservation [7]. The work in [7] suggests that research with a simple rational model on energy conservation decision-making is needed for both organisational and residential sectors.

Our focus, in this paper, is to import ideas from discrete choice theory and statistical physics to develop models for energy conservation decision-making. The interest here is a model that will capture how individual's choices about energy conservation are influenced by their socioeconomic attributes and interaction. By socioeconomic attributes, we are referring to individual's gender, income level, religion, and ethnicity.

3.2. Discrete Choice with Social Interaction. We propose, in this section, a discrete choice with social interaction model for energy conservation behaviour. The discussion here follows closely the presentations in [20,21]. Imagine there are $\mathrm{N}$-energy users who are to decide on whether to conserve or not to conserve energy at some common time. We encode the choice made by the $i^{\text {th }}$ energy consumer as $\sigma_{i} \in\{-1,1\}$, with $\sigma_{i}=1$ representing conservation decision and $\sigma_{i}=-1$ representing nonconservation decision. The decisions of all the consumers in the reference group is an $\mathrm{N}$-coordinate vector $\sigma=\left(\sigma_{1}, \cdots, \sigma_{N}\right)$. In the sequel we denote by $\sigma_{-i}=\left(\sigma_{1}, \cdots, \sigma_{i-1}, \sigma_{i+1}, \cdots, \sigma_{N}\right)$, the $(N-1)$-dimensional vector of decisions made by all consumers less the decision of consumer $i$. From an economic perspective, consumer $i$ will decide on $\sigma_{i}$ if it maximizes his/her utility function $V\left(\sigma_{i}\right)$ expressed as

$$
V\left(\sigma_{i}\right)=u\left(\sigma_{i}\right)+S\left(\sigma_{i}, \mu^{e}\left(\sigma_{-i}\right)\right)+\varepsilon\left(\sigma_{i}\right)
$$

The quantities $u\left(\sigma_{i}\right)$ and $S\left(\sigma_{i}, \mu^{e}\left(\sigma_{-i}\right)\right)$ are, respectively, referred to as the private and social utilities/incentives the $i^{\text {th }}$ consumer derives from the decision $\sigma_{i}$. The perception/belief of consumer $i$ about the choices $\sigma_{-i}$ made by the rest of the consumers in the reference group is quantified by the probability $\mu^{e}\left(\sigma_{-i}\right)$. Further, $\varepsilon\left(\sigma_{i}\right)$ is that part of the consumer's utility that is unobserved by the experimenter/modeller, but consumer $i$ is aware of it at the time of taking the decision $\sigma_{i}$. It is referred to as the random part of the utility. For our application of the discrete choice theory to energy use behaviour, the random utility may correspond to the consumer's inability to properly optimize their utility. This may result in the consumer making incorrect choice even when he/she is provided with full and complete information. Further, it may reflect the lack of information on the part of the consumer about new technologies.

The authors of [22] noted that the above discrete choice model is equivalent to a statistical mechanical model called the Curie-Weiss model when we have the following: 
(i) $u\left(\sigma_{i}\right)=h \sigma_{i}$, for some $h \in \mathbb{R}$. For this choice of the private utility $h=1 / 2[u(1)-u(-1)]$, i.e., $h$ is proportional to the difference between the private utilities of the two choices.

(ii) $S\left(\sigma_{i}, \mu^{e}\left(\sigma_{-i}\right)\right)=J \sigma_{i} m$, where $m=1 / N-1 \sum_{j \neq i} \sigma_{i}$ and $J \in \mathbb{R}$. This choice of our social utility belongs to a large class utility functions that exhibit totalistic and constant strategic complementarity [23].

(iii) $\varepsilon(-1)-\varepsilon(1)$ is logistically distributed, i.e.,

$$
\mathbb{P}(\varepsilon(-1)-\varepsilon(1) \leq x)=\frac{1}{1+\exp (-\beta x)}, \beta>0
$$

It follows from the above considerations that if consumer $i$ decides on $\sigma_{i}=1$, then it is the case that $V(1)>V(-1)$. It follows from here that the probability that consumer $i$ takes the decision $\sigma_{i}$ is given by

$$
P\left(\sigma_{i}\right)=\frac{\exp \left(\beta\left[h \sigma_{i}+J \sigma_{i} \bar{m}_{i}^{e}\right]\right)}{\sum_{\eta_{i} \in\{-1,1\}} \exp \left(\beta\left[h \eta_{i}+J \eta_{i} \bar{m}_{i}^{e}\right]\right)} .
$$

The parameter $h$ quantifies an individual's personal motivation to make a choice, and $J$ modulates an individual's motivation to conform (not to conform) to social norm $m$. Positive $h$ values indicate that at the private level an individual will be motivated to choose 1 , and the alternative choice of -1 will be preferred for negative $h$ values. $J>0$ on the other hand, will promote conformity to social norm and nonconformity to social norm if $J<0$. The parameter $\beta$ describes how much of the actual decision of the consumer is determined by the deterministic part of the utility.

Under the assumption that energy consumers in a reference group do not communicate or coordinate their decisions, the probability that the reference group makes a choice $\sigma=\left(\sigma_{1}, \cdots, \sigma_{N}\right)$ is given by

$$
\begin{aligned}
P(\sigma) & =\frac{\exp \left(\beta \sum_{i=1}^{N}\left[h \sigma_{i}+J \sigma_{i} m\right]\right)}{\sum_{\eta_{1} \in\{-1,1\}} \cdots \sum_{\eta_{N} \in\{-1,1\}} \exp \left(\beta \sum_{i=1}^{N}\left[h \eta_{i}+J \eta_{i} m\right]\right)} \\
& =\prod_{i=1}^{N} \frac{\exp \left(\beta\left[h \sigma_{i}+J \sigma_{i} m\right]\right)}{\sum_{\eta_{i} \in\{-1,1\}} \exp \left(\beta\left[h \eta_{i}+J \eta_{i} m\right]\right)}
\end{aligned}
$$

Note from (8) that $\sigma_{1}, \cdots, \sigma_{N}$ are independent and identically distributed Bernoulli random variables with common mean

$$
E\left(\sigma_{1}\right)=\tanh (\beta h+\beta J m)
$$

The strong law of large numbers then tells us that as $N$ tends to infinity the empirical average $m_{N}=1 / N \sum_{i=1}^{N} \sigma_{i}$ almost surely tends to tanh $(\beta h+\beta J m)$. Further, it follows from [24] that the fix point equation (10) below has at least one solution:

$$
m^{*}=\tanh \left(\beta h+\beta J m^{*}\right) \text {. }
$$

Under the social equilibrium condition of $[25,26]$, we have that

$$
E\left(\sigma_{1}\right)=m=\tanh (\beta h+\beta J m) .
$$

This implies that the equilibrium mean choice level of a consumer in the reference group is a solution to (10). Under the social equilibrium condition, the discrete choice model under consideration is equivalent to the Currie-Weiss model [27]. This statistical mechanical model could exhibit multiple equilibria due to the properties of the tanh function. The multiplicity of mean equilibrium levels of choice depends sensitively on the strength of the social utility and the magnitude of the bias towards one choice induced by the private utility. In particular, the following result is proved in.

Proposition 1. For the solution(s) to (10) the following holds:

(1) If $h=0$, then (10) has

(a) a unique solution for $0<\beta J \leq 1$

(b) three solutions for the case $\beta J>1$

(2) Suppose $h \neq 0$, then there is a threshold $H$, depending on $\beta$ and $h$, such that (10) has

(a) a unique solution with the same sign as $h$ if $|\beta h|$ $>H$

(b) three solutions, one of them has the same sign as $h$, and the others have opposite sign, if $|\beta h| \leq H$

3.3. Discrete Choice and Energy Use Behaviour Models. In the sociopsychological approach to modelling behaviour, beliefs are the building blocks. The beliefs about an object are formed base on an individual's evaluation of the attributes of the object [10]. How this evaluation of attributes of an object is done is silent in the sociopsychological models. This evaluation, we believe, is the starting point of the discrete choice theory. Here, it is natural to assume that individuals evaluate the attributes of an object based on their own attributes and the influences from their respective social environments. Thus, individuals' attributes and social environments are the determinants of their evaluation of attributes of an object, which in turn inform their beliefs about the object. In particular, the objects forming the basis of the sociopsychological model are obtained from the discrete choice model after optimizing the utility function of the discrete choice model. Let us now look at how the various models for energy use behaviour fit into the present scheme of discrete choice with social interaction.

The discrete choice model offers a natural extension to the theory of reasoned action. In that, upon optimizing the 
utility function, the optimal utility is still a random quantity, the optimal private utility now becomes the attitude and the optimal social utility becomes the social norm part of behaviour. Here, we are looking at the theory of reasoned action with random behaviour. The randomness here could be modelling some intrinsic attributes of the individual that cannot be observed by the modeller. Due to this random nature of behaviour, we can no longer describe behaviour with a value but rather with probability distribution on the set of all possible realisations of behaviour.

The theory of planned behaviour extends the theory of reasoned action by adding perceived behavioural control. People can control their behaviour if they know themselves very well. It is this knowledge that determines the level of control an individual will have over his or her behaviour. In the context of the discrete choice theory, this control may refer to the extent to which individuals know about the parameters $h$ and $\beta$, i.e., how individual evaluates their beliefs and the motivation for the individual to comply or not to comply with the perceived evaluation by the rest of the reference group. Thus, to get the discrete choice analogue of the theory of planned behaviour, the parameters $\beta$ and $h$ need to be taken randomly with some a priori distribution, which could be updated as and when we get new information via Bayes rule. This leads us into disordered discrete choice models. For a statistical mechanical version of this model, we refer the reader to [28]. We shall not treat these class of models here.

The discrete choice analogue for the normative and gain goal frames are the case where the social utility component of the utility dominates the private utility of an individual. Over here, one is preoccupied with the needs of neighbourhood or the environment. The hedonic goal frame corresponds to the case where the private utility part of an individual's utility dominates. We shall later on observe that the logistic model considered in [14] results from the discrete choice model when there is no social utility.

The random component of the utility function for the discrete choice theory may corresponds to the individual's inability to properly optimize their utility. This may result in the individual making incorrect choice even when he/she is provided with full and complete information. Further, it may reflect the lack of information on the individual's part about new technologies. This connects the discrete choice theory to the energy technologists' model.

3.4. The Curie-Weiss Model. This section is devoted to the statistical mechanical model that will be shown to be equivalent to the above discrete choice model. We will follow closely the discussion and notation from [21]. The objects appearing in the discrete choice theory are obtained for the statistical mechanical model after taking the thermodynamic limit, i.e., the limit as the number of consumers tend to infinity. The statistical mechanics model of interest is referred to as the Curie-Weiss model is introduced as follows: consider a positive integer $n$ and let $\Omega_{n}=\{-1,+1\}^{n}$, be the set of spin configurations of the system. The Curie-Weiss model is given by an energy function/Hamiltonian $H_{n}$ on $\Omega_{n}$, which for any $\sigma \in \Omega_{n}$ takes the form

$$
H_{n}(\sigma)=-\frac{J}{2 n} \sum_{i, j=1}^{n} \sigma_{i} \sigma_{j}-h \sum_{i=1}^{n} \sigma_{i}=-n\left[\frac{J}{2} m_{n}^{2}+h m_{n}\right]-\frac{1}{2},
$$

where

$$
m_{n}=\frac{1}{n} \sum_{i=1}^{n} \sigma_{i}
$$

The quantity $m_{n}$ is called the empirical average, and it assumes values from the set $\{-1,-(n-1) / n, \cdots, 0, \cdots,(n$ $-1) / n, 1\}$, for each positive integer $n$. The parameter $h$ and $J$ are real valued, and they represent the interaction bias and interaction strength, respectively. $h$ is the strength of external field, and $J$ measures the strength of the interaction among pairs of spins. The first term in the Hamiltonian turns to align pairs of spins if $J>0$ and the pairs will not be aligned if $J<0$. The second term also tends to align spins in the direction of the external field $h$. Let $\alpha$ be the uniform probability measure on $\{-1,1\}$, i.e., $\alpha(-1)=\alpha(1)=1 / 2$ and put $P_{n}=$ $\alpha^{\otimes n}$, the product probability measure on $\Omega_{n}$. The above Hamiltonian defines a physical system whose equilibrium state is given by the probability measure

$$
\mu_{n}(\sigma)=\frac{1}{Z_{n}} \exp \left(-\beta H_{n}(\sigma)\right) P_{n}(\sigma), \quad \sigma \in \Omega_{n},
$$

The normalization term $Z_{n}$ is called the partition function of the model and is defined as

$$
Z_{n}=\sum_{\tilde{\sigma} \in \Omega_{n}} \exp \left(-\beta H_{n}(\tilde{\sigma})\right) P_{n}(\tilde{\sigma})
$$

The parameter $\beta$ is the inverse temperature. The CurieWeiss Hamiltonian can be written in terms of the empirical average as follows:

$$
H_{n}(\sigma)=F\left(m_{n}\right)=\frac{J m_{n}^{2}}{2}+h m_{n}+o(n)
$$

where $m_{n}$ is the empirical measure of $\sigma$.

Further, we write the $\mu_{n}$ expectation for any bounded measurable function $f$ defined on $\Omega_{n}$ as follows:

$$
<f>_{n, \beta, h}=\int_{\Omega_{n}} f\left(m_{n}\right) d \mu_{n}(\sigma) .
$$

The specific empirical magnetization of the model is also given as

$$
m_{n}(\beta, h)=\frac{1}{n} \int_{\Omega_{n}}\left(\sum_{i=1}^{n} \sigma_{i}\right) d \mu_{n}(\sigma)=\frac{1}{\beta n} \frac{\partial}{\partial h} \log Z_{n} .
$$


The second equality follows from (12), (14), and (15). The specific magnetization then becomes

$$
m(\beta, h)=\lim _{n \rightarrow \infty} m_{n}(\beta, h)
$$

To understand the form of the specific magnetization, the exponential growth rate of the partition function $Z_{n}$ will be crucial. Let us now lay some foundation to access this growth rate. Suppose $n=n_{1}+n_{2}$ and recall from (13) that

$$
m_{n}=\frac{n_{1}}{n} m_{n_{1}}+\frac{n_{2}}{n} m_{n_{2}}
$$

Using the convexity of the map $x \mapsto x^{2}$ and (12), we get

$$
Z_{n} \leq Z_{n_{1}} Z_{n_{2}}
$$

$$
\lim _{n \rightarrow \infty}\left\langle f>_{n, \beta, h}=\left\{\begin{array}{l}
f(z(\beta, h)) \\
\frac{1}{2} f(z(\beta,+))+\frac{1}{2} f(z(\beta,-))
\end{array}\right.\right.
$$

where $z(\beta, h)$ is the global minimizer of the function

$$
\begin{aligned}
i_{\beta, h}(z)= & -\frac{1}{2} \beta J z^{2}-\beta h z+\frac{1-z}{2} \log (1-z) \\
& +\frac{1+z}{2} \log (1+z)
\end{aligned}
$$

on $|z| \leq 1$ and $z(\beta, \pm)=\lim _{h \rightarrow 0^{ \pm}} z(\beta, h)$.

(2) The specific magnetization $m(\beta, h)$ of the CurieWeiss model is given by the unique global minimizer $z(\beta, h)$ of (25) for $\beta>0, h \neq 0$ and $0<\beta J \leq 1, h=0$. In particular,

$$
\begin{aligned}
m(\beta, \pm) & =\lim _{h \rightarrow 0^{ \pm}} m(\beta, h) \\
& = \begin{cases}z(\beta, 0)=0 & \text { for } 0<\beta J \leq 1, \\
z(\beta, \pm) \neq 0 & \text { for } \beta J>1 .\end{cases}
\end{aligned}
$$

The above theorem says that whenever $\beta J>1$, a dramatic change will be observed in the macroscopic behaviour of the system if a small change is made in $h$ around the value $h=0$. Observe that the specific magnetization for the Curie-Weiss model, given in the above theorem, coincides with some of the social equilibrium average choice levels found as solution to the self-consistency equation (10). Because the selfconsistency equation (10) holds for the global minimizers of (25). Some of the solutions to the self-consistency equation
Therefore

$$
\log Z_{n} \leq \log Z_{n_{1}}+\log Z_{n_{2}}
$$

It follows from the Fekete's subadditive lemma [29] that

$$
\lim _{n \rightarrow \infty} \frac{1}{n} \log Z_{n}, \quad \text { existsand } \lim _{n \rightarrow \infty} \frac{1}{n} \log Z_{n}=\inf _{n} \frac{1}{n} \log Z_{n} \text {. }
$$

Next, we state a result taken from [24] that characterizes the limiting $\mu_{n}$ expectation of bounded measurable functions of the empirical average $m_{n}(\beta, h)$.

\section{Theorem 2.}

(1) Suppose $f$ is a bounded and continuous function from $\mathbb{R}$ to $\mathbb{R}$. Then,

for $\beta>0, h \neq 0$ and $0<\beta J \leq 1, h=0$

for $\beta J>1, h=0$,

(10) may not be specific magnetization of the Curie-Weiss model as they may be local minimizer and local maximizer of the function (25). In particular, for the case $h \neq 0$, Theorem 2 suggests that there is a unique specific magnetization that has the same sign as $h$ as opposed to the result in Proposition 12 , where it is observed that there are three self-consistent solutions when $\beta$ is high and $h \neq 0$. In fact, the selfconsistent solution that has the same sign as $h$ becomes the specific magnetization for the Curie-Weiss model, and the other two are, respectively, local minimum and maximum of the function (25). The local maximum and minimum are, respectively, the unstable and metastable self-consistent mean choice levels of a consumer. For the rest of the paper, we will concentrate on the self-consistent solutions that are consistent with the statistical mechanical prescription, i.e., global minimizers of (25). Thus, up to these self-consistent mean choice levels, the discrete choice model and the Curie-Weiss model are equivalent. In particular, the joint distribution (8) of the decisions made by the consumers in the reference group is obtained from the Curie-Weiss model by taking $n$-dimensional marginal of the Curie-Weiss Gibbs measure $\mu_{n}$, given in (14), as $n$ tends to infinity.

\section{Multipopulation Curie-Weiss Model}

We present a multipopulation version of the Curie-Weiss model in this section. This model will be our benchmark model for modelling energy conservation behaviour of energy users. Here, we follow closely the discussion in [30]. Our aim is to understand the collective behaviour of consumers in a 
reference group from the intricate interactions among the consumers [20, 22, 31]. The statistical mechanical model introduced in this section will help to achieve our aim. Already statistical mechanics has successfully been applied to study the aggregate behaviour of individuals facing interdependence binary choice [27]. Statistical mechanics provides a general framework for understanding aggregate behaviours of interacting individuals. Here, our emphasis is on using a multipopulation Curie-Weiss model to study energy conservation behaviour of a community of energy users who are interacting with themselves and their environment.

The Hamiltonian $H_{n}$ for configurations (collective decisions) $\sigma \in\{-1,1\}^{n}$ given by

$$
H_{n}(\sigma)=\frac{1}{2 n} \sum_{i, l=1}^{n} J_{i l} \sigma_{i} \sigma_{l}+\sum_{i=1}^{n} h_{i} \sigma_{i}
$$

is made up of the interaction part controlled by the interaction strengths $J_{i l}$ and the external field part also modulated by $h_{i}$. The interactions tend to align spins/decisions $\sigma_{i}$ and $\sigma_{l}$ whenever $J_{i l}>0$, i.e., neighbouring consumers will prefer to be either +1 (conserve energy) or -1 (not to conserve energy). Negative interaction coupling strengths, i.e., $J_{i l}<0$, will result in neighbouring consumers taking different decisions. From statistical mechanics literature, the parameter $h_{i}$ is referred to as the external magnetic field applied to the site $i$. From our socioeconomic applications point of view, $h_{i}$ is the intrinsic attributes of a consumer that determines his/her behaviour. Positive values for $h_{i}$ favours +1 decision while negative values of $h_{i}$ favours -1 decision. For social science applications of the model of interest, we refer the reader to $[20,27,30]$.

Here, we study the energy conservation behaviour of energy consumers in a reference group, and how the behaviour of an individual in the reference group is influenced by his/her intrinsic socioeconomic attributes and social interaction with the rest of the members of the reference group. Notable socioeconomic attributes of energy consumers may include gender, place of residence, level of education, ethnicity, beliefs, values, and norms. These attributes influence a consumer's evaluation of the attributes of energy alternatives and energy saving technologies. A key assumption that we would employ in our study is that consumers with the same socioeconomic attributes will tend to behave the same way, while consumers with different socioeconomic attributes behave differently. This assumption reduces the number of parameters in the Hamiltonian (27), allowing a systematic statistical estimation procedure to be developed to estimate the parameters of the model from data.

Suppose our reference group is made up of energy consumers with $k$ socioeconomic attributes, and the group is made up on $n$ consumers. Therefore, consumer $i$ will be identified by his/her attributes vector

$$
a_{i}=\left(a_{i}^{(1)}, a_{i}^{(2)}, \cdots, a_{i}^{(k)}\right)
$$

Here, $a_{i}^{(j)} \in A_{j}$ is the $j^{\text {th }}$ attribute of individual $i$, and $A_{j}$ is the set of attribute alternatives for attribute $j=1, \cdots, k$. Let $d(j)=\left|A_{j}\right|$ be the number of attribute alternatives for attribute $j$, which we assume to be finite. Therefore, our reference group of size $n$ can be partitioned into

$$
d=\prod_{j=1}^{k} d(j)
$$

nonover lapping subgroups, with each subgroup made up of individuals with the same socioeconomic attribute alternatives. Suppose we identify these sub-groups with number $1,2, \cdots, d$, we denote by $I_{n_{g}}$ and $\left|I_{n_{g}}\right|=n_{g}$ the subset of individuals in sub-group $g$ and the number of individuals in sub-group $g$, respectively. Thus, $n=n_{1}+\cdots+n_{d}$ and for any $g \neq g^{\prime}=1,2, \cdots, d, I_{n_{g}} \cap I_{n_{g^{\prime}}}=\varnothing$. Further, we assume that for each $g=1, \cdots, d$,

$$
\gamma_{n}^{g}=\frac{n_{g}}{n} \text { and } \gamma^{g}=\lim _{n \rightarrow \infty} \gamma_{n}^{g}
$$

We assume that all individuals in subgroup $g$ have the same private utility $\bar{h}_{g}$ and for any pair of subgroups $g$ and $g^{\prime}, J_{i l}=\bar{J}_{g g^{\prime}}$ whenever $i \in I_{n_{g}}$ and $l \in I_{n_{g^{\prime}}}$. It implies from this assumption and equation (27) that

$$
\begin{aligned}
H_{n}(\sigma)= & \frac{1}{2 n} \sum_{g=1}^{d} \sum_{g^{\prime}=1}^{d}\left(\sum_{i \in I_{n_{g}}} \sum_{l \in I_{n_{g^{\prime}}}} J_{i l} \sigma_{i} \sigma_{l}\right) \\
& +\sum_{g=1}^{d} \sum_{i \in I_{n_{g}}} h_{i} \sigma_{i}=\frac{1}{2 n} \sum_{g=1}^{d} \sum_{g^{\prime}=1}^{d} \bar{J}_{g g^{\prime}} n_{g^{\prime}} m_{n}^{g} n_{g^{\prime}} m_{n}^{g^{\prime}} \\
& +\sum_{g=1}^{d} \bar{h}_{g} n_{g^{\prime}} m_{n}^{g}=n\left[\sum_{g=1}^{d} \sum_{g^{\prime}=1}^{d} \frac{\bar{J}_{g g^{\prime}}}{2} \gamma_{n}^{g} m_{n}^{g} \gamma_{n}^{g^{\prime}} m_{n}^{g^{\prime}}\right. \\
& \left.+\sum_{g=1}^{d} \bar{h}_{g} \gamma_{n}^{g} m_{n}^{g}\right]=n\left[\sum _ { g = 1 } ^ { d } m _ { n } ^ { g } \left(\sum_{g^{\prime}=1}^{d} \frac{\bar{J}_{g g^{\prime}}}{2} \gamma_{n}^{g} \gamma_{n}^{g^{\prime}} m_{n}^{g^{\prime}}\right.\right. \\
& \left.\left.+\bar{h}_{g} \gamma_{n}^{g}\right)\right]=n \sum_{g=1}^{d} m_{n}^{g} U_{n, g} .
\end{aligned}
$$

In the above, we have used that

$$
m_{n}^{g}=\frac{1}{n_{g}} \sum_{i \in I_{n_{g}}} \sigma_{i}
$$

is the empirical average decision for the individuals in subgroup g. The Hamiltonian of the model has now become a function of the empirical averages of the decisions of individuals in the subgroups. From our socioeconomic application of the model, $H_{n}$ measures the level of satisfaction for each joint decision vector of the individuals in the reference group. Here, $\bar{J}_{g_{g^{\prime}}}$ is the strength of the influence subgroup $g$ 


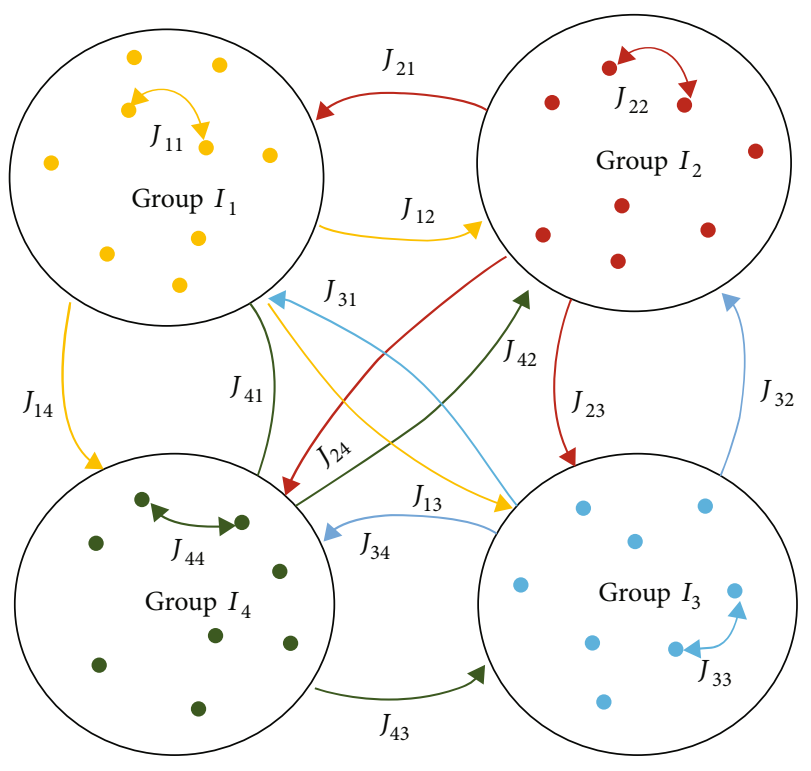

FIGURE 1: A representation of the interaction network for the model $\left(I_{1}, I_{2}, I_{3}\right.$, and $I_{4}$ are the subgroups of the reference group. Within $I_{1}$ (resp. $I_{2}, I_{3}, I_{4}$ ), individuals feel a mean-field interaction with coupling $J_{11}$ (resp. $J_{22}, J_{33}$, and $J_{44}$ ). Subgroup $I_{1}$ influences the other subgroups with coupling strength $\left(J_{12}, J_{13}, J_{14}\right)$.

has on sub-group $g^{\prime}$. It is also a measure of the social incentive for subgroups $g$ and $g^{\prime}$ to interact. Positive $\bar{J}_{g g^{\prime}}$ values imply that the subgroups are satisfied if their empirical means have the same signs. On the other hand, if the $\bar{J}_{g g^{\prime}}<0$, the empirical means of the different subgroups will prefer to have different signs, i.e., interaction between the two subgroups is discouraged. Figure 1 presents an artistic impression of the $\bar{J}_{g g^{\prime}}$ 's when $d=4$. The parameter $\bar{h}_{g}$ is the private incentive of the group $g$. This describes how subgroup $g$ is satisfied with itself.

The finite volume Gibbs measure associated to our multipopulation Hamiltonian $H_{n}(31)$ takes the form

$$
\omega_{n}(\sigma)=\frac{e^{H_{n}(\sigma)} P_{n}(\sigma)}{Z_{n}}, \quad \text { for } \quad \sigma \in \Omega_{n}
$$

where

$$
Z_{n}=\sum_{\tilde{\sigma} \in \Omega_{n}} e^{H_{n}(\tilde{\sigma})} P_{n}(\tilde{\sigma})
$$

is the normalization constant called the partition function of the model.

Recall from (32) the definition of the subgroup empirical average $m_{n}^{g}$ and write $m_{n}=\left(m_{n}^{1}, \cdots, m_{n}^{d}\right) \in[-1,1]^{d}$ for the vector of subgroup empirical averages. Define for any $n \in \mathbb{N}$,

$$
F_{n}\left(m_{n}\right)=\sum_{g=1}^{d} \sum_{g^{\prime}=1^{d}} \frac{\bar{J}_{g g^{\prime}}}{2} \gamma_{n}^{g} \gamma_{n}^{g^{\prime}} m_{n}^{g} m_{n}^{g^{\prime}}+\sum_{g=1}^{d} \bar{h}_{g} \gamma_{n}^{g} m_{n}^{g} .
$$

Suppose a sequence $\left(m_{n}\right)_{n \geq 1}$ of empirical mean vectors converges to $m \in[-1,1]^{d}$, then

$$
\begin{aligned}
F(m)= & \sum_{g=1}^{d} \sum_{g^{\prime}=1}^{d} \frac{\bar{J}_{g g^{\prime}}}{2} \gamma^{g} \gamma^{g^{\prime}} m^{g} m^{g^{\prime}} \\
& +\sum_{g=1}^{d} \bar{h}_{g} \gamma^{g} m^{g}=\lim _{n \rightarrow \infty} F_{n}(m) .
\end{aligned}
$$

In the sequel, we shall write $\gamma, \bar{h}$, and $\bar{J}$ for

$$
\begin{aligned}
\gamma & =\left(\gamma^{1}, \cdots, \gamma^{d}\right), \bar{h}=\left(\bar{h}^{1}, \cdots, \bar{h}^{d}\right) \in \mathbb{R}^{d} \text { and } \bar{J} \\
& =\left(\bar{J}_{g, g^{\prime}}\right)_{1 \leq g, g^{\prime} \leq d} .
\end{aligned}
$$

The finite volume pressure of the multipopulation model then becomes

$$
p_{n}=\frac{1}{n} \log Z_{n}
$$

The large $n$ behaviour of the model is controlled by the exponential growth rate of the partition function, i.e., the pressure. It follows from [32] that the thermodynamic limit

$$
p(\gamma, \bar{h}, \bar{J})=\lim _{n \rightarrow \infty} \frac{1}{n} \log Z_{n}
$$

exists. An earlier proof of this claim for the case $k=1$ and $\gamma_{n}^{g}=\gamma^{g}$, for all $n$, is found in $[33,34]$.

Theorem 3. Suppose the parameters, $\gamma, h$, and $\bar{J}$, are given as above. Then the limiting pressure has the following variational representation

$$
p(\gamma, \bar{h}, \bar{J})=\sup _{m \in[-1,1]^{d}}\left[F(m)-\sum_{g=1}^{d} \gamma^{g} I\left(m^{g}\right)\right],
$$

with

$$
I\left(m^{g}\right)=\frac{1-m^{g}}{2} \log \left(1-m^{g}\right)+\frac{1+m^{g}}{2} \log \left(1+m^{g}\right)
$$

and $F$ is defined in (36).

The maximizers $m$ of the variational problem (40) have coordinates $\mathrm{m}^{g}$ satisfying the self-consistency equations

$$
m^{g}=\tanh \left(U_{g}\right), \quad g=1,2, \cdots, d .
$$

Here, we have used that $U_{g}$ is the limit of $U_{n, g}(31)$ as $n$ tends to infinity, i.e.,

$$
U_{g}=\sum_{g^{\prime}=1^{d}} \frac{\bar{J}_{g g^{\prime}}}{2} \gamma^{g} \gamma^{g^{\prime}} m^{g^{\prime}}+\bar{h}_{g} \gamma^{g} .
$$


In the sequel, we put $J_{g g^{\prime}}=\left(\bar{J}_{g g^{\prime}} / 2\right) \gamma^{g} \gamma^{g^{\prime}}$ and $h_{g}=\gamma^{g} \bar{h}_{g}$. Therefore, it follows from equation (43) that

$$
U_{g}=\sum_{g^{\prime}=1}^{d} J_{g g^{\prime}} m^{g^{\prime}}+h_{g}, \text { for } \quad g=1, \cdots, d
$$

The above theorem is proved in [35]. The proof for the case $k=1$ and $\gamma_{n}^{g}=\gamma^{g}$ for all $n$ was earlier given in [33]. It follows from $[33,35]$ and references therein that the marginal distribution for the $i^{\text {th }}$ energy user in subgroup $g$ to make a choice $\sigma_{i} \in\{-1,+1\}$ is given by

$$
\mathbb{P}\left(\sigma_{i}\right)=\frac{e^{\sigma_{i} U_{g}}}{e^{U_{g}}+e^{-U_{g}}} .
$$

The expected value of the $i^{\text {th }}$ individual's decision results in the self-consistency equations

$$
\begin{aligned}
m^{g} & =\mathbb{E}\left(\sigma_{i}\right)=\frac{e^{U_{g}}}{e^{U_{g}}+e^{-U_{g}}}-\frac{e^{-U_{g}}}{e^{U_{g}}+e^{-U_{g}}} \\
& =\tanh \left(U_{g}\right), \text { for } \quad g=1,2, \cdots, d .
\end{aligned}
$$

Recall from (46) that $m^{g}$ is the average decision of the individuals in group $g$. Again, with respect to the parameters $J_{g g^{\prime}}$ and $h_{g}$, it follows from (44) that $U_{g}$ is a linear regression model with covariates $m^{g^{\prime}}$, for $g^{\prime}=1, \cdots, d$. Further, we model the private utility part $h_{g}$ as a linear regression of the attributes alternatives (28) as follows:

$$
h_{g}=\alpha_{0}+\sum_{j=1}^{k} \sum_{s=1}^{d(j)} \alpha_{s}^{j} \sum_{r \in L} \delta_{a_{g}^{j}, r} .
$$

Recall that our model considers $k$ socioeconomic attributes. Here, $\alpha_{s}^{j}$ measures the value an individual places on the $s$ attribute alternative of attribute $j$, and $\alpha_{0}$ is the uniform private utility for all the decision makers. $a_{g}^{j}$ is the alternative of attribute $j^{\text {th }}$ present in group $g$, and $d(j)$ is the count of the different alternative for attribute $j$. The set of all alternatives for the different attributes is the alternatives set $L$, and $\delta_{a_{q}^{j}, r}$ is the Kronecker delta function. The form of our private utility $h_{g}(47)$ accounts for the influence of each of the attribute alternatives on the private utility contrary to the case considered in [34], where the influence of some of the attribute alternatives was put to zero. Consequently, the linear regression models for the $U_{g}$ have $J_{g g^{\prime}}, \alpha_{s}^{j}$, and $\alpha_{0}$ as their parameters that should be estimated.

The case $J_{g g^{\prime}}=0$ for every $g$ and $g^{\prime}$ is the model with no interaction. The regression model for $U_{g}$ becomes

$$
U_{g}=h_{g}, \text { for } g=1, \cdots, d .
$$

Therefore, it follows from (45) that the probability for the $i^{\text {th }}$ individual in subgroup $g$ to decide on $\sigma_{i}=1$ takes the form

$$
\mathbb{P}\left(\sigma_{i}=1\right)=\frac{e^{U_{g}}}{e^{U_{g}}+e^{-U_{g}}}=\frac{1}{1+e^{-2 U_{g}}} .
$$

Our model reduces to the logistic regression when the $J_{g g^{\prime}}=0$ for every $g$ and $g^{\prime}$. Therefore, taking logarithm of the quotient of the probabilities to choose $\sigma_{i}=1$ against $\sigma_{i}=-1$ results in the following interaction version of the logistic regression

$$
\frac{1}{2} \log \left(\frac{\mathbb{P}\left(\sigma_{i}=1\right)}{\mathbb{P}\left(\sigma_{i}=-1\right)}\right)=\sum_{g^{\prime}=1}^{d} J_{g g^{\prime}} m_{g^{\prime}}+h_{g} .
$$

As mentioned above, if $J_{g g^{\prime}}=0$ for every $g$ and $g^{\prime}$, the above logarithm of the quotient becomes the logistic regression model outlined in Section 2.4. This model was used in [14] to study the energy conservation behaviour of energy users.

4.1. Parameter Estimation. In what follows, we will estimate $m^{g}$ (46) by $m_{n}^{g}$ (32). For large enough $N$, $\gamma_{n}^{g}$ will be close to $\gamma^{g}$; hence, we drop the $n$ dependence of $\gamma_{n}^{g}$. Consequently, the parameters $J_{g g^{\prime}}$ and $h_{g}$ become $n$ independent. Two procedures will be discussed for the estimation of the parameters.

4.1.1. Estimation Procedure 1. Recall from (46) that tanh $\left(U_{g}\right)$ model's prediction of $m^{g}$ and it is estimated by the empirical average choice $m_{n}^{g}$. Due to the monotonicity property of the tanh function, we estimate the parameters of the model via looking for the parameter values that minimizes the sum of squares error

$$
\sum_{g}\left[\arctan h\left(m_{n}^{g}\right)-U_{g}\right]^{2}
$$

since the parameters to be estimated are linear in $U_{g}$. This procedure is only possible if $m_{n}^{g}$ stays away from being +1 or -1 . In the regime where the interaction strength is nonvanishing the covariates of the regression model may be correlated. Due to this, the partial least squares (PLS) estimation procedure may be used [36-40]. This procedure extracts a set of independent predictors from a set of correlated predictors that jointly explain most of the variability in both the dependent and the independent variables. The key question here is, how many independent predictors should we use for the estimation? This question is answered with the help of the root mean square error of prediction for models formed from increasing subsets of the extracted independent predictors that are sorted in decreasing order of importance. The root mean square error of prediction for the models decreases initially the more predictors are introduced one at a time. The best fitted model is the one beyond which the root mean square error of prediction starts to rise. One could not access confidence intervals for PLS estimates. Bootstrap resampling 
techniques are usually applied to the best fitted model to generate a number of estimates that are used to construct confidence intervals for the estimates.

4.1.2. Estimation Procedure 2. This section outlines the parameter estimation procedure for multipopulation CurieWeiss model $[41,42]$. Recall from the expression for $U_{g}$ in (44) and (31) that

$$
\begin{aligned}
H_{n}(\sigma) & =n \sum_{g=1}^{d} m^{g} U_{g}=n \sum_{g=1}^{d} m^{g}\left(\sum_{g^{\prime}=1}^{d} J_{g g^{\prime}} m^{g^{\prime}}+h_{g}\right) \\
& =n\left(\sum_{g, g^{\prime}=1}^{d} J_{g g^{\prime}} m^{g} m^{g^{\prime}}+\sum_{g=1}^{d} h_{g^{\prime}} m^{g}\right) \\
& =n(\langle J m, m\rangle+\langle h, m\rangle)
\end{aligned}
$$

where $m=\left(m^{1}, \cdots, m^{d}\right), h=\left(h_{1}, \cdots, h_{d}\right)$ and $J$ is the interaction matrix

$$
J=\left(\begin{array}{cccc}
J_{11} & J_{12} & \cdots & J_{1 d} \\
J_{12} & J_{22} & \cdots & J_{2 d} \\
\vdots & \vdots & & \vdots \\
J_{1 d} & J_{2 d} & \cdots & J_{d d}
\end{array}\right)
$$

From the self-consistency equations (46) we have that

$$
\begin{aligned}
& m^{g}(J, h)=\mathbb{E}\left(\sigma_{i}\right)=\tanh \left(\sum_{g^{\prime}=1}^{d} J_{g g^{\prime}} m^{g^{\prime}}+h_{g}\right) \\
& \qquad \text { for } g=1,2, \cdots, d .
\end{aligned}
$$

If the self-consistency equations above admit a unique thermodynamic stable solution $m(J, h)=\left(m^{1}(J, h), \cdots, m^{d}\right.$ $(J, h))$, then the following identity hold [33, 42],

$$
\lim _{n \rightarrow \infty} \omega_{n}\left(m_{n}^{g}\right)=m^{g}(J, h), \quad g=1, \cdots, d
$$

where $\omega_{n}$ and $m_{n}^{g}$ are defined as in (33) and (32), respectively. Note that $\omega_{n}\left(m_{n}^{g}\right)$ is the average value of the magnetization. According to [42], the susceptibility matrix of the model has the following entries:

$$
\begin{aligned}
\chi_{g g^{\prime}} & =\lim _{n \rightarrow \infty} \frac{\partial}{\partial h_{g^{\prime}}} \omega_{n}\left(m_{n}^{g}\right), \quad \text { for } g, g^{\prime}=1, \cdots, d \\
& =\frac{\partial m^{g}(J, h)}{\partial h_{g^{\prime}}}=\frac{\partial}{\partial h_{g^{\prime}}}\left(\tanh \left(h_{g}+\sum_{g^{\prime}=1}^{d} J_{g g^{\prime}} m^{g^{\prime}}\right)\right) \\
& =\left(1-\left(m^{g}\right)^{2}\right)\left(\delta_{g g^{\prime}}+\sum_{g^{\prime}=1}^{d} J_{g g^{\prime}} \chi_{g g^{\prime}}\right),
\end{aligned}
$$

where $\delta_{g g^{\prime}}=1$ if $g=g^{\prime}$ and zero otherwise is the Kronecker delta. Hence, the susceptibility matrix is given as,

$$
\chi=P(I+J \chi)
$$

where $\mathbf{P}=\operatorname{diag}\left\{1-\left(m^{1}\right)^{2}, \cdots, 1-\left(m^{d}\right)^{2}\right\}$ and $I$ is an identity matrix. Again, for each pair of groups $g, g^{\prime}=1$, $\cdots, d$,

$$
\begin{aligned}
\frac{\partial}{\partial h_{g^{\prime}}} \omega_{n}\left(m^{g}(\sigma)\right) & =\frac{\partial}{\partial h_{g^{\prime}}}\left(\frac{\sum_{\sigma \in \Omega_{n}} m^{g} e^{H_{n}(\sigma)}}{\sum_{\sigma \in \Omega_{n}} e^{H_{n}(\sigma)}}\right) \\
& =n_{g^{\prime}}\left(\omega_{n}\left(m^{g} m^{g^{\prime}}\right)-\omega_{n}\left(m^{g}\right) \omega_{n}\left(m^{g^{\prime}}\right)\right) .
\end{aligned}
$$

It follows from (56) and (58) that the susceptibility matrix $\chi$ can be computed with the help of (57). With this, we obtain an expression for the interaction matrix $J$, which is related to the averages and correlations of the magnetizations in the thermodynamic limit [42], i.e.,

$$
J=\left(P^{-1}-\chi^{-1}\right)
$$

The external field $h$ is obtained by using the above estimate for the interaction matrix $J$ and the self-consistency equations (54), i.e.,

$$
\begin{aligned}
& h_{g}=\tanh ^{-1}\left(m^{g}(J, h)\right)-\sum_{g^{\prime}=1}^{d} J_{g g^{\prime}} m^{g^{\prime}}(J, h) \\
&=\tanh ^{-1}\left(\lim _{n \rightarrow \infty} \omega\left(m^{g}\right)\right)-\sum_{g^{\prime}=1}^{d} J_{g g^{\prime}} \lim _{n \rightarrow \infty} \omega\left(m^{g^{\prime}}\right), \\
& \text { for } \quad g=1, \cdots, d .
\end{aligned}
$$

Upon estimating the $h_{g}$ 's, we then fit the following regression model (47) to determine the socioeconomic attributes that contribute to the estimated private incentive $h_{g}$ for each of the groups.

$$
h_{g}=\alpha_{0}+\sum_{j=1}^{k} \sum_{s=1}^{d(j)} \alpha_{s}^{j} \sum_{r \in L} \delta_{a_{g}^{j}, r} .
$$

\section{Conclusion}

This study incorporates ideas from economics, energy management, social psychology, discrete choice theory, and statistical physics. In the sociopsychological models discussed, it was noted that beliefs are their building blocks and that beliefs about an object are formed base on an individual's evaluation of the attributes of the object $[10,13]$. How these evaluations are made, we argue, is the starting point of the discrete choice model. The study proposes a statistical mechanical model to bridge the sociopsychological, economics, and energy models used in energy management. 
Our model is motivated by the equivalence between discrete choice theory with social interaction and the CurieWeiss model from statistical mechanics [22]. This formulation provides a general framework for understanding how interdependences modulate the aggregate behaviours of individuals.

\section{Data Availability}

No data were used to support this study.

\section{Conflicts of Interest}

The authors declare that they have no conflicts of interest.

\section{Acknowledgments}

The authors thank the staff at the Mathematics and Statistics Department and Energy and Engineering Department of University of Energy and Natural Resources for their support and kindness during the period this paper was written.

\section{References}

[1] E. N. Kumi, The Electricity Situation in Ghana: Challenges and Opportunities. CGD Policy Paper, Center for Global Development, Washington DC, 2017, http://www.cgdev. org/publication/electicity-situation-ghana-challenges-andopportunities.

[2] C. Wilson and H. Dowlatabadi, "Models of decision making and residential energy use," Annual Review of Environment and Resources, vol. 32, no. 1, pp. 169-203, 2007.

[3] S. Gyamfi, S. Krumdieck, and T. Urmee, "Residential peak electricity demand response-highlights of some behavioural issues," Renewable and Sustainable Energy Reviews, vol. 25, pp. 71-77, 2013.

[4] A. H. Sanstad and R. B. Howarth, "Consumer rationality and energy efficiency," in Proceedings of the ACEEE 1994 Summer Study on Energy Efficiency in Buildings, Washington D.C., 1994.

[5] T. G. Cowing and D. L. McFadden, Microeconomic Modeling and Policy Analysis: Studies in Residential Energy Demand, Academic Press, Orlando, Florida, 1984.

[6] S. Gyamfi, "Demand response assessment and modelling of peak electricity demand in the residential sector: information and communication requirements," $\mathrm{PhD}$ Thesis, University of Canterbury, Department of Mechanical Engineering, 2010.

[7] L. Lutzenhiser, "Social and behavioral aspects of energy use," Annual Review of Energy and the Environment, vol. 18, no. 1, pp. 247-289, 1993.

[8] E. Aronson, "Energy use: the human dimension," in Committee on Behavioral and Social Aspects of Energy Consumption and Production, National Research Council, Freeman, New York, 1984.

[9] P. C. Stern, Improving Energy Demand Analysis. Panel on Energy Demand Analysis, Committee on Behavioral and Social Aspects of Energy Consumption and Production, National Research Council, National Academy Press, Washington, DC, 1984.

[10] I. Ajzen, Attitudes, Personality and Behaviour, Open University Press, Milton Keynes, Second edition, 1988.
[11] D. Trafimow, "The theory of reasoned action," Theory \& Psychology, vol. 19, no. 4, pp. 501-518, 2009.

[12] G. A. Miller, "The magical number seven plus or minus two: some limits on our capacity for processing information," Psychological Review, vol. 63, no. 2, pp. 81-97, 1956.

[13] I. Ajzen, "The theory of planned behavior," Organizational Behavior and Human Decision Processes, vol. 50, no. 2, pp. 179-211, 1991.

[14] U. Bariss, A. Dandens, L. Timma, A. Blumberga, and D. Blumberga, "How to assess involvement of electricity end user in energy efficiency improvement - analysis of survey results," Energy Procedia, vol. 72, pp. 270-277, 2015.

[15] S. Lindenberg and L. Steg, "Normative, gain and hedonic goal frames guiding environmental behavior," Journal of Social Issues, vol. 63, no. 1, pp. 117-137, 2007.

[16] L. Steg, J. W. Bolderdijk, K. Keizer, and G. Perlaviciute, “An integrated framework for encouraging pro-environmental behaviour: the role of values, situational factors and goals," Journal of Environmental Psychology, vol. 38, pp. 104-115, 2014.

[17] J. M. Nolan, P. W. Schultz, R. B. Cialdini, N. J. Goldstein, and V. Griskevicius, "Normative social influence is underdetected," Society for Personality and Social Psychology, vol. 34, no. 7, pp. 913-923, 2008.

[18] M. Tonglet, P. S. Phillips, and M. P. Bates, "Determining the drivers for householder pro-environmental behaviour: waste minimisation compared to recycling," Resources, Conservation and Recycling, vol. 42, no. 1, pp. 27-48, 2004.

[19] E. Vìgants, A. Blumberga, L. Timma, I. İjabs, and D. Blumberga, "The dynamics of technological substitution: the case of ecoinnovation diffusion of surface cleaning products," Journal of Cleaner Production, vol. 132, pp. 279-288, 2016.

[20] W. A. Brock and S. N. Durlauf, "Discrete choice with social interactions," The review of Economic Studies, vol. 68, no. 2, pp. 235-260, 2001.

[21] A. A. Opoku, K. O. Edusei, and R. K. Ansah, "A conditional Curie Weiss model for stylized multi-group binary choice with social interaction," Journal of Statistical Physics, vol. 171, no. 1, pp. 106-126, 2018.

[22] S. N. Durlauf, "How can statistical mechanics contribute to social science?," Proceedings of the National Academy of Sciences of the United States of America, vol. 96, no. 19, pp. 10582-10584, 1999.

[23] R. Cooper and A. John, "Coordinating coordination failures in Keynesian models," The Quarterly Journal of Economics, vol. 103, no. 3, pp. 441-463, 1988.

[24] R. S. Ellis, Entropy, Large Deviations and Statistical Mechanics, Classics in Mathematics, Springer, 1985.

[25] C. Manski, "Identification problems in the social sciences," in Sociological Methodology, P. Marsden, Ed., vol. 23, Basil Blackwell, Cambridge, 1993.

[26] C. F. Manski, "Identification of endogenous social effects: the reflection problem," Review of Economic Studies, vol. 60, no. 3, pp. 531-542, 1993.

[27] S. N. Durflar, Statistical Mechanics Approach to Socioeconomic Behavior, vol. 203 of NBER, technical working paper series, technical working paper, 1996.

[28] A. Bovier and V. Gayrard, "The thermodynamics of the CurieWeiss model with random couplings," Journal of Statistical Physics, vol. 72, no. 3-4, pp. 643-664, 1993. 
[29] M. Fekete, "Über die Verteilung der Wurzeln bei gewissen algebraischen Gleichungen mit ganzzahligen Koeffizienten," Mathematische Zeitschrift, vol. 17, no. 1, pp. 228-249, 1923.

[30] A. A. Opoku, G. Osabutey, and C. Kwofie, "Parameter evaluation for a statistical mechanical model for binary choice with social interaction," Journal of Probability and Statistics, vol. 2019, Article ID 3435626, 10 pages, 2019.

[31] D. Mcfadden, "Economic choices," The American Economic Review, vol. 91, no. 3, pp. 351-378, 2001.

[32] A. A. Opoku and G. Osabutey, "A multi-population CurieWeiss model for binary choice with social interaction," in preparation.

[33] I. Gallo and P. Contucci, "Bipartite mean field spin systems," in Existence and Solution, vol. 14, MPEJ, 2008.

[34] I. Gallo, "An equilibrium approach to modelling social interaction," PhD Thesis, University of Bologna, Bologna, Italy, 2009.

[35] A. A. Opoku and G. Osabutey, "Multipopulation spin models: a view from large deviations theoretic window," Journal of Mathematics, vol. 2018, Article ID 9417547, 13 pages, 2018.

[36] H. Abdi, "Partial least squares regression and projection on latent structure regression (PLS Regression)," WIREs Computational Statistics, vol. 2, no. 1, pp. 97-106, 2010.

[37] K. S. Ng, A Simple Explanation of Partial Least Squares, Draft, 2013.

[38] H. Wold, "Estimation of principal components and related models by iterative least squares," in Multivariate Analysis, $\mathrm{P}$. R. Krishnaiaah, Ed., pp. 391-420, Academic Press, New York, 1966.

[39] S. Wold, "Personal memories of the early PLS development," Chemometrics and Intelligent Laboratory Systems, vol. 58, no. 2, pp. 83-84, 2001.

[40] K. J. Worsley, "An overview and some new developments in the statistical analysis of PET and fMRI data," Human Brain Mapping, vol. 5, no. 4, pp. 254-258, 1997.

[41] R. Burioni, P. Contucci, M. Fedele, C. Vernia, and A. Vezzani, "Enhancing participation to health screening campaigns by group interactions," Scientific Reports, vol. 5, no. 1, article 9904, 2015.

[42] M. Fedele, C. Vernia, and P. Contucci, "Inverse problem robustness for multi-species mean-field spin models," Journal of Physics A: Mathematical and Theoretical, vol. 46, no. 6, article $065001,2013$. 